\title{
Bericht zur Prüfung im Oktober 2003 über Mathematik der Lebensversicherung (Grundwissen)
}

Jürgen Strobel (Köln) and Hans-Jochen Bartels (Mannheim)

Blätter der DGVFM Band XXVI, Heft 3 (Mai 2004), 511 - 516.

\section{Aufgabe (25 Punkte)}

$$
\begin{gathered}
f_{0} B-a_{0} V_{1}=c_{0} \\
f_{t} B+V_{t}-a_{t} V_{t+1}=c_{t} \text { für } t=1, \ldots, n-2
\end{gathered}
$$

und

$$
f_{n-1} B+V_{n-1}=c_{n-1}
$$

sei das einen Versicherungsvertrag beschreibende lineare Gleichungssystem mit:

- $0<a_{t}<1$ für $t=0,1, \ldots, n-2$,

- den Beitragszahlungsmodalitäten $f_{0}, f_{1}, \ldots f_{n-1}$,

- den Zahlen $c_{t}>0 \quad t=1, \ldots n-1$, welche die Leistungen des Tarifs beschreiben, und

- den zugehörigen Deckungsrückstellungen $V_{t}$ für $t=1, \ldots, n-1$.

Es gelte $f_{n-1} B=c_{n-1}$ und $f_{t} B<c_{t}$ für $t=0,1,2, . ., m$ mit $m<n-2$ und $f_{t} B>c_{t}$ für $t=m+1, \ldots, n-2$.

Man zeige: Mindestens $m+1$ Deckungsrückstellungen in der Folge $V_{1}, V_{2}, \ldots, V_{n-1}$ sind negativ.

\section{Lösung}

Aus $f_{0} B=c_{0}+a_{0} V_{1}$ folgt wegen $f_{0} B<c_{0}$ die Beziehung $V_{1}<0$, und hieraus ergibt sich rekursiv aus den angegebenen Bedingungen wegen $f_{t} B-c_{t}+V_{t}=a_{t} V_{t+1}$ und $a_{t}>0$ sofort: $V_{2}, V_{3}, \ldots, V_{m+1}<0$ und damit insgesamt die Behauptung. Es gilt ferner $V_{n-1}=0$, wegen $f_{n-2} B+V_{n-2}=c_{n-2}$ sowie $f_{n-2} B>c_{n-2}$ folgt hieraus $V_{n-2}<0$. Rekursiv folgt dann sogar $V_{n-3}, \ldots, V_{m+2}<0$.

In Worten formuliert heißt dies, daß man durch nicht ausreichende Prämien zu Beginn negative Deckungskapitale aufbaut, danach - d.h. nach dem Jahr $m$ - werden die negativen Deckungskapitale zwar wieder aufgefüllt, bleiben aber noch negativ, bis schließlich $V_{n-1}=0$ wird und damit letztlich die Äquivalenzgleichungen erfüllt werden. Solche Tarifgestaltungen sind auf jeden Fall zu vermeiden, da negative Deckungsrïckstellungen im Stornofall zu Verlusten des Versicherungsunternehmens führen und aus Sicht des Kunden einen zu billigen Versicherungsschutz bieten (bei gezieltem Storno zum richtigen Zeitpunkt). 